\title{
An Examination of Rostral Anterior Cingulate Cortex Function and Neurochemistry in Obsessive-Compulsive Disorder
}

\author{
Brian P Brennan*,1,2,3, Olga Tkachenko ${ }^{4}$, Zachary J Schwab ${ }^{4}$, Richard J Juelich ${ }^{5}$, Erin M Ryan', Alison J Athey', \\ Harrison G Pope ${ }^{1,3}$, Michael A Jenike ${ }^{2,3}$, Justin T Baker ${ }^{3,5}$, William DS Killgore ${ }^{3,4,6}$, James I Hudson ${ }^{1,3}$, \\ J Eric Jensen ${ }^{3,7}$ and Scott L Rauch ${ }^{3,4}$ \\ 'Biological Psychiatry Laboratory, McLean Hospital, Belmont, MA, USA; 2Obsessive-Compulsive Disorders Institute, McLean Hospital, Belmont, MA, \\ USA; ${ }^{3}$ Department of Psychiatry, Harvard Medical School, Boston, MA, USA; ${ }^{4}$ Social, Cognitive, and Affective Neuroscience Laboratory, McLean \\ Hospital, Belmont, MA, USA; ${ }^{5}$ Psychotic Disorders Division, McLean Hospital, Belmont, MA, USA; ${ }^{6}$ Department of Psychiatry, University of Arizona \\ College of Medicine, Tucson, AZ, USA; ${ }^{7}$ McLean Imaging Center, McLean Hospital, Belmont, MA, USA
}

\begin{abstract}
The anterior cingulate cortex is implicated in the neurobiology of obsessive-compulsive disorder (OCD). However, few studies have examined functional and neurochemical abnormalities specifically in the rostral subdivision of the ACC (rACC) in OCD patients. We used functional magnetic resonance imaging ( $\mathrm{fMRl}$ ) during an emotional counting Stroop task and single-voxel J-resolved proton magnetic resonance spectroscopy ( $\mathrm{H}-\mathrm{MRS}$ ) in the rACC to examine the function and neurochemistry of the rACC in individuals with $O C D$ and comparison individuals without OCD. Between-group differences in rACC activation and glutamine/glutamate ratio (Gln/Glu), Glu, and Gln levels, as well as associations between rACC activation, Gln/Glu, Glu, Gln, behavioral, and clinical measures were examined using linear regression. In a sample of 30 participants with $O C D$ and 29 age- and sex-matched participants without OCD, participants with OCD displayed significantly reduced rACC deactivation compared with those without OCD in response to OCD-specific words versus neutral words on the emotional counting Stroop task. However, Gln/Glu, Glu, and Gln in the rACC did not differ between groups nor was there an association between reduced rACC deactivation and Gln/Glu, Glu, or Gln in the OCD group. Taken together, these findings strengthen the evidence for AACC dysfunction in OCD, but weigh against an underlying association with abnormal rACC glutamatergic neurotransmission.

Neuropsychopharmacology (20I5) 40, I866-I876; doi:I0.1038/npp.20I5.36; published online 4 March 2015
\end{abstract}

\section{INTRODUCTION}

The anterior cingulate cortex (ACC) is a component of the dysfunctional cortico-striatal-thalamo-cortical (CSTC) brain circuitry thought to underlie obsessive-compulsive disorder (OCD) (Saxena and Rauch, 2000). Within the ACC, the dorsal subdivision (dACC; operationally defined as the region of the ACC bounded anteriorly by a coronal plane at the genu of the corpus callosum; Rauch et al, 2003) is thought to mediate cognitive processes, whereas the rostral subdivision ( $\mathrm{rACC}$ ) is thought to mediate affect (Bush et al, 2000; Vogt et al, 1995). The rACC exhibits strong connectivity to other brain regions implicated in OCD (Alexander et al, 1986), and functional magnetic resonance imaging (fMRI) has demonstrated abnormal rACC activation in OCD patients (Fitzgerald et al, 2005, 2010). However, the neurochemical substrates of rACC dysfunction in OCD remain unclear.

*Correspondence: Dr BP Brennan, McLean Hospital, I 15 Mill Street, Belmont, MA 02478, USA, Tel: +| 617855 291।, Fax: + 617855 3585, E-mail: bbrennan@partners.org

Received 10 September 2014; revised 12 January 2015; accepted 13 January 2015; accepted article preview online 9 February 2015
CSTC neuronal pathways generally utilize the excitatory neurotransmitter glutamate (Glu) (Pittenger et al, 2011), and magnetic resonance spectroscopy (MRS) studies have implicated glutamatergic dysfunction in OCD (Gnanavel et al, 2013; Rosenberg et al, 2000, 2004; Yücel et al, 2008). Most MRS studies have quantified Glu together with glutamine (Gln) as a composite measure labeled 'Glx', making it difficult to interpret the relative contributions of Gln and Glu (Brennan et al, 2013). However, advanced MRS techniques, such as $J$-resolved proton MRS ( $\left.{ }^{1} \mathrm{H}-\mathrm{MRS}\right)$ (Jensen et al, 2009), can measure Glu and Gln separately, thus permitting calculation of the Gln/Glu ratio, which reflects flux through the Glu-Gln cycle-a potentially better gauge of glutamatergic neurotransmission (Brennan et al, 2010; Igarashi et al, 2001; Iltis et al, 2009; Öngür et al, 2008, 2011).

To further elucidate the functional and neurochemical abnormalities in the rACC in OCD, we combined an fMRI probe of rACC function, the emotional counting Stroop paradigm (ecStroop) (Whalen et al, 1998), with J-resolved ${ }^{1} \mathrm{H}$-MRS to measure rACC Glu and Gln levels in patients with OCD versus individuals without OCD. Given evidence of ACC hyperactivation in OCD from studies using functional neuroimaging both at rest (Baxter et al, 1987; Machlin et al, 1991; Perani et al, 1995; Swedo et al, 1989) and 
with symptom provocation (Adler et al, 2000; Breiter et al, 1996; Mataix-Cols et al, 2004; Rauch et al, 1994), plus evidence that glutamatergic neurotransmitter activity is coupled with neuronal glucose metabolism (Sibson et al, 1998), we hypothesized that: (1) OCD patients would demonstrate increased rACC activation compared with the non-OCD group for OCD-specific versus neutral words on the ecStroop; (2) OCD patients would demonstrate an elevated rACC Gln/Glu ratio compared with the non-OCD group; and (3) rACC activation and Gln/Glu would be significantly correlated in OCD patients.

\section{PATIENTS AND METHODS}

\section{Participant Selection}

OCD patients $\geq 18$ years of age, right-handed, with a DSM-IV primary diagnosis of $\mathrm{OCD}$, and scoring $\geq 18$ on the YaleBrown Obsessive-Compulsive Scale (Y-BOCS) were recruited from the Obsessive-Compulsive Disorders Institute at McLean Hospital, an intensive residential treatment program for severe OCD. All OCD participants received MRI scans within the first week of admission (most within 2-4 days of admission) and before medication changes or clinically significant behavioral therapy. Exclusion criteria included: history of schizophrenia, bipolar disorder, Tourette's syndrome, or an autism-spectrum disorder; substance abuse or dependence (with the exception of nicotine) within 3 months of enrollment; hoarding as the primary OCD symptom; history of significant organic brain disease; significant medical illness; current pregnancy or lactating; or MRI contraindication. Participants taking medications that principally modulate the glutamate system (eg, memantine, riluzole, lamotrigine, topiramate, $N$-acetylcysteine) were excluded. All other psychiatric medications were permitted if at a stable dose for $\geq 4$ weeks before scanning.

Community individuals without OCD, $\geq 18$ years of age, right-handed, with no DSM-IV psychiatric diagnosis, taking no psychoactive medications, and with no psychiatric illness among first-degree relatives were recruited by advertising. Exclusion criteria included: positive urine screen for drugs of abuse before MRI scan; significant neurologic or medical illness; current pregnancy or lactating; or MRI contraindication.

\section{Clinical Evaluation}

After obtaining informed consent, approved by the McLean Hospital Institutional Review Board, the following data were collected by the study physician (BPB or HGP): demographic information, medical/psychiatric history, diagnoses via the Structured Clinical Interview for DSM-IV (SCID), OCD symptom information via the Yale-Brown Obsessive-Compulsive Checklist (Y-BOCS-CL), OCD symptom severity via the Y-BOCS, and depression severity via the MontgomeryAsberg Depression Rating Scale (MADRS). Based on responses on the Y-BOCS-CL, a predominant symptom dimension was determined for each OCD participant using the method described by Landeros-Weisenberger et al, 2010). In general, collection of clinical data occurred between 1 and 3 days before MRI scanning. However, because of scheduling difficulties, four OCD participants could not be scanned until day 6 after collection of clinical data.

\section{Functional Task}

Participants performed a version of the ecStroop paradigm modified for OCD. The ecStroop was developed as a variant of the traditional emotional color-naming Stroop task that requires a motor response (button press) instead of a spoken response in order to minimize head movement during fMRI (Whalen et al, 2006). Prior studies using the ecStroop task have shown it to be a useful probe of rACC function in both healthy volunteers (Whalen et al, 1998) and patients with anxiety disorders (Britton et al, 2009; Shin et al, 2001). During each trial, participants viewed a set of 1-4 identical words displayed simultaneously on a screen for $1.5 \mathrm{~s}$, counted the number of words, and pressed a button corresponding to that number (see details in Supplementary Figure S1). In separate conditions, participants viewed three different types of words: (1) neutral words (eg, mailbox, garage, telephone), (2) general negative words unrelated to OCD (eg, tragedy, contempt, poverty), and (3) OCD-specific words reflecting several OCD symptom dimensions (eg, knife, guilty, filthy, mistake). Words in the three conditions were matched for length, part of speech, and frequency of usage.

Immediately after scanning, participants rated the word stimuli according to relevance $(0=$ low to $6=$ high $)$, arousal $(0=$ low to $6=$ high $)$, and valence $(-3=$ negative to $+3=$ positive $)$.

\section{Image Acquisition}

All imaging was conducted on a Siemens Trio scanner (Siemens Medical Solutions USA, Malvern, PA) with a 32 channel Trans-Imaging Matrix platform upgrade located at the McLean Imaging Center and operating at a 3-Tesla magnetic field strength. All participants underwent a routine anatomic scan to screen for structural abnormalities.

Functional MRI images (blood oxygenation level dependent (BOLD)) were acquired using a gradient echo $\mathrm{T}^{*}$ weighted sequence (TR/TE/flip angle $=2 \mathrm{~s} / 30 \mathrm{~ms} / 90^{\circ}$ ). The $\mathrm{T} 1, \mathrm{~T} 2$, and gradient-echo functional images were collected in the same plane (24 coronal slices angled perpendicular to the AC-PC line) with the same slice thickness $(7 \mathrm{~mm}$, skip $1 \mathrm{~mm}$; voxel size $3.5 \times 3.5 \times 8 \mathrm{~mm}$ ), excitation order (interleaved), and phase encoding (foot to head).

MRS acquisition used a modified ${ }^{1} \mathrm{H}$-MRS protocol similar to that described in our previous studies (Brennan et al, 2010; Jensen et al, 2009; Öngür et al, 2008, 2011). Briefly, a $2 \times 2 \times$ $2 \mathrm{~cm}$ voxel was placed on the rACC midsagittally, anterior to the genu of the corpus callosum (Figure 1). Shimming of the voxel was done using a machine automated shimming routine. Following automated optimization of water suppression, carrier frequency, tip angles, and coil tuning, a modified $J$-resolved protocol (2D-JPRESS) was used. The 2D-JPRESS sequence collected 22 TE-stepped spectra with the echo-time ranging from 35 to $350 \mathrm{~ms}$ in $15 \mathrm{~ms}$ increments $(\mathrm{TR}=2 \mathrm{~s}, \mathrm{f} 1$ acquisition bandwidth $=67 \mathrm{~Hz}$, spectral bandwidth $=2 \mathrm{kHz}$, readout duration $=512 \mathrm{~ms}, \mathrm{NEX}=16 / \mathrm{TE}$-step, approximate scan duration $=12 \mathrm{~min}$ ) providing enough $J$-resolved bandwidth $(67 \mathrm{~Hz})$ to resolve Glu and Gln (Figure 1).

\section{Statistical Analyses}

Behavioral measures. For each word type, we calculated participants' accuracy and average response time on correct 


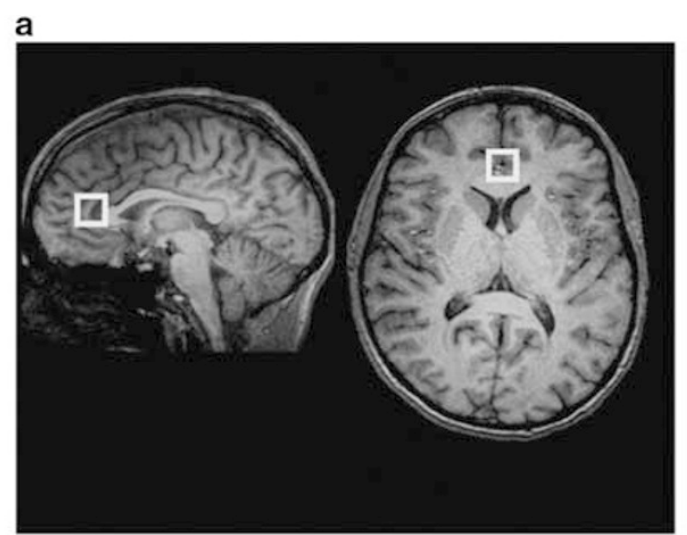

b

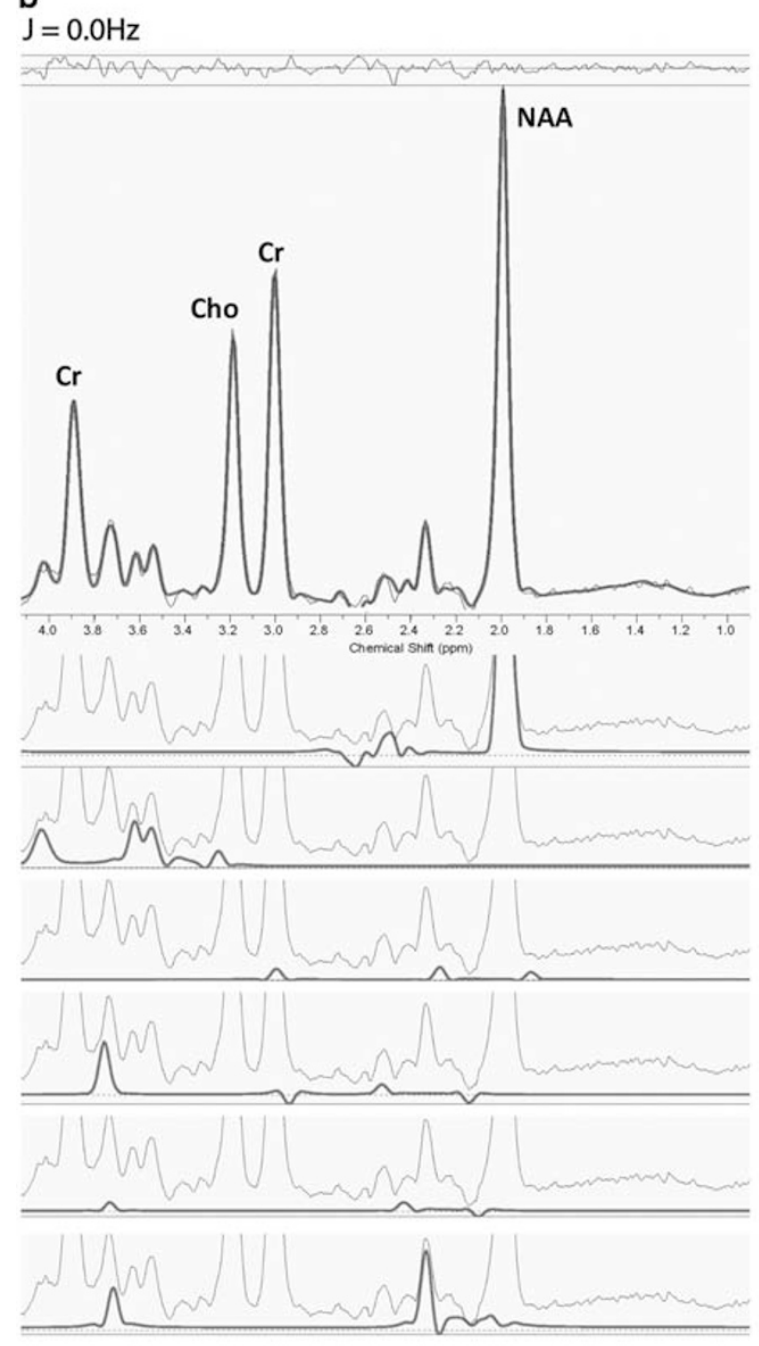

$$
\mathrm{J}=6.9 \mathrm{~Hz}
$$

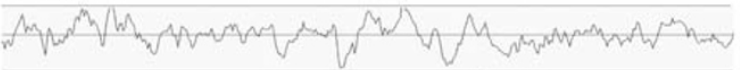
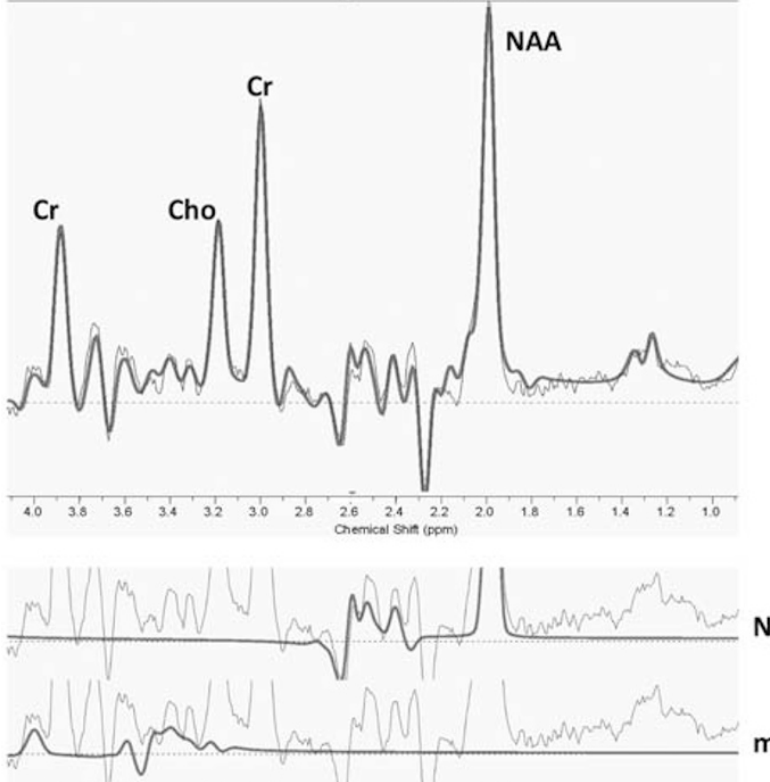
$\mathrm{ml}$

GABA

GSH

GIn

Glu

Figure I (a) Sagittal and axial brain slices showing placement of magnetic resonance spectroscopy (MRS) voxel in the rostral anterior cingulate cortex (rACC). (b) Sample rACC MRS spectral extractions from the 2D J-resolved data set depicting the theoretically modeled LCModel fits for J $=0.0 \mathrm{~Hz}$ and $J=6.9 \mathrm{~Hz}$, each shown with total LCModel fit, residual and major J-coupled subcomponents. Both extractions provide illustration of our quantitative method for on and off-axis spectra. As described in the Patients and Methods, all 64 J-resolved spectral extractions are quantified in this manner. Cho, choline; Cr, total creatine; GABA, $\gamma$-aminobutyric acid; Gln, glutamine; Glu, glutamate; GSH, glutathione; ml, myo-inositol; NAA, N-acetylaspartate. 
trials. The Stroop interference effect represented the difference in response time between OCD-specific and neutral words. We used linear regression to examine the association of group and word type with behavioral measures and postscan ratings, as well as between-group differences in Stroop interference. The $\alpha$-value was set at 0.05 , two tailed. All analyses were performed using Stata 9.2 software.

fMRI. Data were preprocessed and analyzed in SPM8 with slice-time correction. Acquired images were realigned and unwarped, co-registered to each individual's anatomical image, spatially normalized to match the template of the Montreal Neurological Institute (MNI), and spatially smoothed (full width half maximum $=6 \mathrm{~mm}$ ) using a $3 \mathrm{D}$ Gaussian filter. Scans were inspected using the Artifact Detection Tool (http://www.nitrc.org/projects/artifact_de tect/) and volumes exceeding $3 \mathrm{SD}$ in mean global intensity or $1.0 \mathrm{~mm}$ of interscan motion were regressed out of the analyses. Using a general linear model approach (GLM), the functional data for each participant were convolved to a waveform derived from the experimental design and the canonical hemodynamic response function in SPM8.

A two-step random-effects approach was used for all analyses. First, activation during the conditions of interest was fitted using the general linear model in SPM for each participant individually, yielding statistical parametric maps isolating the activity unique to the condition of interest relative to the activity associated with the control condition. Thus, for each participant, a 'contrast image' was produced that reflected the pattern of BOLD signal change because of the independent variable for each task. Second, these contrast images were entered as the dependent variables in a series of random-effects analyses in SPM, with group assignment as the independent or predictor variables, depending on the analyses. The principal contrasts of interest were the OCDspecific $v s$ neutral and general negative $v s$ neutral conditions. Functional data were analyzed using region of interest (ROI) and whole-brain exploratory approaches. First, an ROI analysis was performed by constructing a region mask of the ACC defined according to the anatomical atlas of Tzourio-Mazoyer et al (2002) using the Wake Forest University PickAtlas Utility (Maldjian et al, 2003). This ROI analysis was carried out at a statistical threshold of $P<0.05$, family-wise error (FWE) corrected. Second, wholebrain analyses were also undertaken using a whole-brain FWE correction of $P<0.05$, unless noted otherwise.

Psychophysiological interaction (PPI) analyses (O'Reilly et al, 2012) were conducted to examine significant differences in task-dependent functional connectivity between groups. Given the putative role of rACC inhibition of amygdalar activity in the resolution of emotional conflict (Etkin et al, 2006), we reasoned that functional connectivity between rACC and amygdala during conditions of emotional conflict might be altered in OCD. A spherical mask with a 5$\mathrm{mm}$ radius was generated around the peak-activated voxel $(10,50,10)$ from the rACC cluster identified in the main between-group task analysis of OCD versus neutral words using the Wake Forest University PickAtlas. A second mask defining the union of the left and right amygdalae was created using the same tool. The two masks were subsequently used to define individual seed regions within a PPI analysis framework adapted from the Generalized PPI Toolbox ((McLaren et al, 2012); https://www.nitrc.org/ projects/gppi). The deconvolved activity time course was extracted for each seed for each participant. A model was created for each seed including the time course of activity, the psychological condition, and the interaction term for each participant. Activity within each seed was then regressed against the interaction term, with the time course and psychological variables serving as regressors of no interest. These results were then entered into a second-level random-effects analysis to examine between-group differences in task-dependent functional connectivity. Because of the small search spaces in the rACC and amygdalae ROIs, this analysis was conducted using a statistical threshold of $P<0.01$, uncorrected.

${ }^{1} H$-MRS. In order to quantify Glu and Gln with the JPRESS data, the 22 TE-stepped free-induction decay series (FIDS) was zero-filled out to 64 points, Gaussian-filtered, and Fourier-Transformed using GAMMA-simulated J-resolved basis sets modeled for $3 \mathrm{~T}$. Every J-resolved spectral extraction within a bandwidth of $67 \mathrm{~Hz}$ was fit with the spectral-fitting package LCModel (Provencher, 1993) and its theoretically correct template. The integrated area under the entire 2D surface for each metabolite was calculated by summing the raw peak areas across all $64 \mathrm{~J}$-resolved extractions for each metabolite as in our prior publications (Brennan et al, 2010; Jensen et al, 2009; Öngür et al, 2008, 2011). Our primary MRS measure, Gln/Glu, is expressed as a ratio, whereas Gln and Glu levels are expressed as a ratio to total creatine $(\mathrm{tCr})$ - a widely used approach to reduce subject-specific variance intrinsic to ${ }^{1} \mathrm{H}$-MRS data (Öngür et al, 2009). In order to examine potential between-group differences in $\mathrm{tCr}$, we compared the $\mathrm{tCr} /$ total signal ratio between groups where total signal is the summation of all fitted raw metabolite peak integrals.

For voxel tissue segmentation, 3D mpRAGE axial image data sets were first converted into NIFTI binary image file format using FSL (FMRIB). FMRIB's Automated Segmentation Tool (FAST; Oxford, UK) was used for tissue segmentation of the T1-weighted image sets into gray matter (GM), white matter (WM), and cerebrospinal fluid (CSF). Partial tissue fractions were then derived and expressed as a percentage of total tissue contribution for the rACC voxel using in-house software. The mean (SD) percentage of GM in the rACC voxel in the non-OCD group and OCD group was $64 \%(1 \%)$ and $64 \%(1 \%)$, WM was $16 \%(1 \%)$ and $14 \%$ (1\%), and CSF was $19 \%(1 \%)$ and $21 \%(1 \%)$.

fMRI ${ }^{1} H$-MRS/behavioral/clinical correlations. The BOLD response signal change (expressed as the first eigenvariate for the BOLD time series) for OCD versus neutral words $(\mathrm{OCD}>$ non-OCD) was extracted for each individual from the rACC cluster maximum $(P<0.05$, FWE corrected) using the MarsBaR toolbox (http://www.marsbar.sourceforge.net/). As our primary statistical approach, we used the cluster maximum for each participant rather than averaging over the entire cluster or over the MRS volume of interest to avoid inclusion of nonsignificant voxels. Secondarily, we performed the same analyses using the cluster average to determine whether this approach yielded similar results. 
The relationships between metabolite levels, BOLD signal change, Stroop interference effect, and Y-BOCS scores were analyzed using linear regression adjusted for age and sex.

\section{RESULTS}

\section{Participants}

A total of 62 participants (31 with OCD and 31 without OCD) completed study procedures. One participant with OCD and three comparison participants were excluded from analysis because of: (1) technical problems (MRS and fMRI data for one OCD participant and MRS data for one comparison participant were lost because of an error in the transfer of data from the MRI scanner); (2) inability to complete scan (one comparison participant requested to stop scanning before complete data could be collected); or (3) conflicting demographic information (one comparison participant was found to have provided discrepant age and date of birth information on prior scans performed at our institution). In addition, behavioral data from one individual with OCD were excluded because of malfunctioning of the button box during administration of the ecStroop task. Demographic and clinical features of the participants are presented in Table 1.

\section{Behavioral}

Participants with OCD had significantly longer response times than comparison participants across all word types (Supplementary Table S1). There was a trend toward greater Stroop interference in the OCD group ( $\beta=9.7$ ( -1.9 to 21.4$)$; $P=0.099$ ). All three postscan ratings showed significant group-by-word type interactions, with participants with OCD rating both general negative and OCD-specific words as significantly more relevant, arousing, and negative than did comparison participants (Supplementary Table S2).

\section{fMRI}

Between group. In our primary between-group ROI analysis, participants with OCD showed rACC hyperactivation compared with non-OCD participants in response to OCD-specific relative to neutral words (MNI coordinates: 10,50,10; $Z=4.10 ; P<0.05$, FWE-corrected; $k=12$; Figure 2a and $b)$. Extraction of signal change relative to baseline fixation from this rACC cluster (Figure 2c) revealed that rACC hyperactivation in participants with OCD reflected an inability to deactivate this region during OCD-specific words. Whole-brain analysis revealed no significant differences for OCD-specific versus neutral words at an FWE correction of $P<0.05$. To detect potentially interesting activation foci for future exploration, we repeated these analyses using a threshold of $P<0.001$, uncorrected, and found hyperactivation in several additional brain regions in OCD participants, including medial prefrontal cortex (mPFC), orbitofrontal cortex (OFC), precuneus, posterior inferior parietal lobule (pIPL), caudate, putamen, amygdala, and insula (Table 2 and Supplementary Figure S2). There were no areas of relative hyperactivation in the non-OCD group for OCD-specific versus neutral words.

For general negative $v s$ neutral words, there were no areas of relative hyperactivation in the OCD or non-OCD group in the ROI or FWE-corrected whole-brain analyses. Supplementary Table S3 shows results for whole-brain analysis using a statistical threshold of $P<0.001$, uncorrected.

Within group. An exploratory within-group ROI analysis showed that participants with OCD exhibited significantly greater rACC activation in response to OCD-specific relative to neutral words (MNI coordinates: $10,50,22 ; Z=4.40$; $P<0.05$, FWE-corrected; $k=18$ ), whereas comparison participants showed no significant difference for this contrast. Whole-brain analysis revealed two additional clusters of activation in the OCD group located in the left and right superior $\mathrm{mPFC}((-12,58,20) ; Z=5.46 ; k=69$ and $(6,58,28)$; $Z=5.08, k=26$, respectively). Repeating these analyses at a threshold of $P<0.001$, uncorrected, we found a larger set of activated regions in the OCD group including $\mathrm{mPFC}$, precuneus, inferior frontal cortex, pIPL, OFC, and insula (Table 3). There were no significant clusters in the non-OCD group at either statistical threshold.

An exploratory analysis of general negative $v s$ neutral words revealed no areas of hyperactivation for either the ROI or whole-brain analyses in either group. Supplementary Table S4 shows results for whole-brain analysis using a statistical threshold of $P<0.001$, uncorrected.

Functional connectivity. We found no significant betweengroup differences in task-dependent functional connectivity between rACC and amygdala in response to OCD versus neutral words.

${ }^{1} H$-MRS. We found no significant differences between $\mathrm{OCD}$ and comparison participants in rACC Gln/Glu ratio or in rACC levels of Glu and Gln separately or the sum of Glu +Gln (Supplementary Table S5 and Supplementary Figure $\mathrm{S} 3)$. We found no significant differences in the $\mathrm{tCr} / \mathrm{total}$ signal ratio $(P=0.83)$ between groups.

\section{fMRI $/{ }^{1} \mathrm{H}-\mathrm{MRS} /$ Behavioral/Clinical Correlations}

The peak rACC BOLD signal change for OCD words versus neutral words showed no significant associations with Gln/ Glu ratio, levels of Glu, Gln or Glu+Gln, Stroop interference, or total Y-BOCS scores in either group. Identical analyses using the rACC BOLD signal change averaged over the entire cluster yielded similar results.

\section{Effects of Potential Confounding Variables}

Comparison of the 6 unmedicated versus the 24 medicated OCD participants using $t$-tests revealed no significant differences in Stroop interference, total Y-BOCS score, peak rACC BOLD activation, Gln/Glu ratio, Glu, Gln, or Glu+Gln (Supplementary Table S6 and Supplementary Figure S4). We also found no significant association between MADRS score and any of these same variables in the OCD group using linear regression adjusted for age and sex (Supplementary Table S7).

\section{DISCUSSION}

To our knowledge, this is the first study to assess the functional and neurochemical integrity of the rACC in patients 
Table I Demographic and Clinical Characteristics

\begin{tabular}{|c|c|c|c|}
\hline Characteristic & $\begin{array}{l}\text { Participants with OCD } \\
\qquad(\mathrm{N}=\mathbf{3 0})\end{array}$ & $\begin{array}{l}\text { Participants without OCD } \\
\qquad(N=29)\end{array}$ & $P$-value \\
\hline Age, years, mean (SD) & $32.0(11.5)$ & $32.4(12.1)$ & $0.91^{a}$ \\
\hline \multicolumn{4}{|l|}{ Sex } \\
\hline Male, N (\%) & $18(60)$ & $13(45)$ & $0.30^{\mathrm{b}}$ \\
\hline Duration of $O C D$, years, mean (SD) & $19.0(12.9)$ & - & \\
\hline Yale-Brown Obsessive-Compulsive Scale, mean (SD) & $27.8(2.6)$ & 0 & \\
\hline Montgomery-Asberg Depression Rating Scale, mean (SD) & $17.1(7.5)$ & $0.66(1.0)$ & \\
\hline \multicolumn{4}{|l|}{ Predominant OCD symptom dimension } \\
\hline \multicolumn{4}{|l|}{ DSM-IV axis I psychiatric comorbidity at the time of study } \\
\hline None, $N(\%)$ & $10(33)$ & - & \\
\hline Major depressive disorder, N (\%) & II (37) & - & \\
\hline Dysthymic disorder, N (\%) & $3(10)$ & - & \\
\hline Panic disorder, $N(\%)$ & $3(10)$ & - & \\
\hline Social anxiety disorder, $N(\%)$ & $3(10)$ & - & \\
\hline Anorexia nervosa, $N(\%)$ & I (3) & - & \\
\hline Bulimia nervosa, $N(\%)$ & I (3) & - & \\
\hline Body dysmorphic disorder, N (\%) & $2(7)$ & - & \\
\hline Trichotillomania, N (\%) & I (3) & - & \\
\hline \multicolumn{4}{|l|}{ Medications at the time of study } \\
\hline Benzodiazepine, $N(\%)^{f}$ & $14(47)$ & - & \\
\hline Other, $N(\%)$ & $4(13)$ & - & \\
\hline Bupropion & $2(7)$ & - & \\
\hline Lithium & I (3) & - & \\
\hline Trazodone ${ }^{g}$ & I (3) & - & \\
\hline Zolpidem ${ }^{g}$ & । (3) & - & \\
\hline Naltrexone ${ }^{g}$ & I (3) & - & \\
\hline \multicolumn{4}{|l|}{ Number of weeks on stable dose of medication } \\
\hline SSRI/SNRI/Clomipramine, mean (SD, range) & $51(109,4-120)$ & - & \\
\hline Second-generation antipsychotic, mean (SD, range) & $35(37,12-108)$ & - & \\
\hline Benzodiazepine, mean (SD, range) & $91(133,8-520)$ & - & \\
\hline Other, mean (SD, range) & $175(232,36-520)$ & - & \\
\hline
\end{tabular}

Abbreviations: OCD, obsessive-compulsive disorder; SNRI, serotonin-norepinephrine reuptake inhibitor; SSRI, selective serotonin reuptake inhibitor.

aby t-test (two tailed).

${ }^{\text {b}}$ By Fisher's exact test (two tailed).

'Fluoxetine $(N=8)$, sertraline $(N=7)$, escitalopram $(N=2)$, and citalopram $(N=1)$.

Venlafaxine $(N=1)$.

eRisperidone $(\mathrm{N}=3)$ and aripiprazole $(\mathrm{N}=3)$.

${ }^{f}$ Clonazepam $(N=7)$, lorazepam $(N=5)$, alprazolam $(N=1)$, and clorazepate $(N=1)$.

UUsed by the same participant. 
a

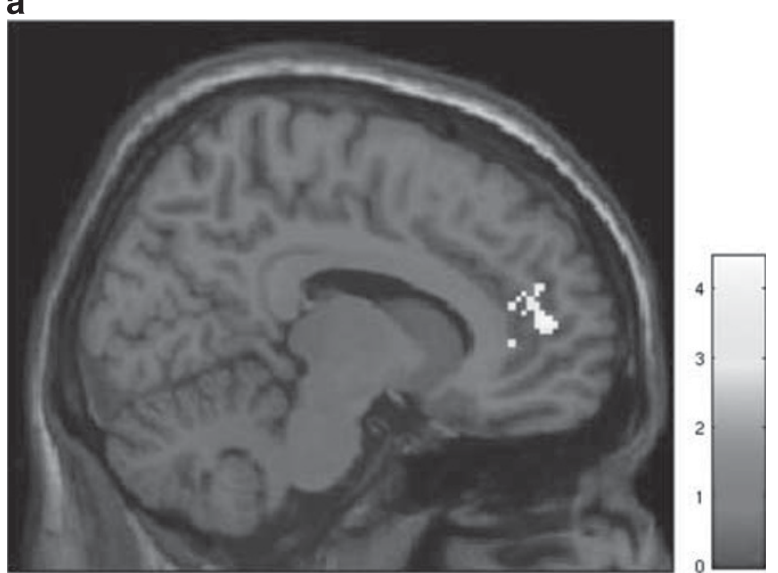

b

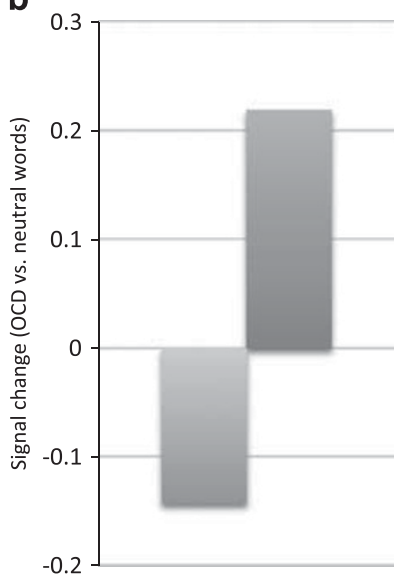

c

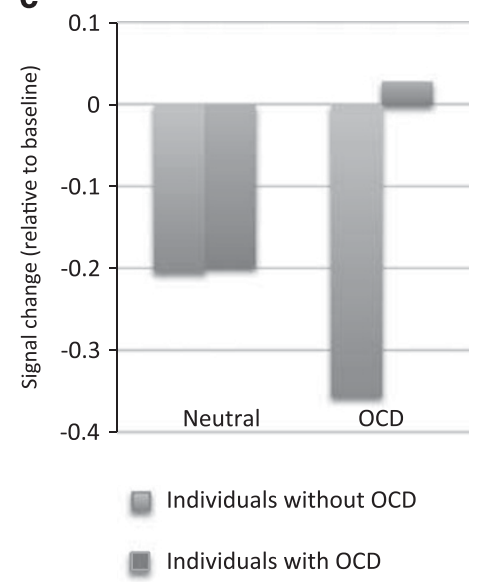

Figure 2 Rostral anterior cingulate ( $\mathrm{rACC}$ ) activation during the emotional counting Stroop task in participants with obsessive-compulsive disorder ( $O C D$ ) and participants without OCD. (a) Between-group difference in activation in rACC for OCD-specific words versus neutral words (OCD participants $>$ non$O C D$ participants). Presented for visualization using a threshold of $P<0.00 \mathrm{I}$, uncorrected. Color bar indicates $t$-values. Signal change values extracted from this rACC cluster ( $P<0.05$, FWE corrected) for (b) OCD-specific words versus neutral words and for (c) individual word types (OCD-specific and neutral) relative to baseline fixation.

with OCD using a multimodal neuroimaging approach combining fMRI and $J$-resolved ${ }^{1} \mathrm{H}$-MRS to separately quantify glutamate and glutamine levels. Using an ecStroop task, we found significantly reduced rACC BOLD deactivation in OCD participants compared with non-OCD participants in response to OCD-specific words versus neutral words. However, we found no difference between groups in rACC Gln/ Glu ratio, and no significant association between rACC BOLD activation and Gln/Glu ratio in the OCD group. Collectively, these findings strengthen the evidence for rACC dysfunction in OCD, but suggest that this dysfunction is not attributable to abnormal rACC glutamatergic neurotransmission.

Our finding of ecStroop-provoked rACC activation is consistent with prior functional neuroimaging studies demonstrating ACC hyperactivity both at rest (Machlin et al, 1991; Perani et al, 1995; Swedo et al, 1989) and with symptom provocation (Adler et al, 2000; Breiter et al, 1996; Mataix-Cols et al, 2004; Rauch et al, 1994) in OCD. However, most of these studies examined the ACC as a single brain region, with only two exceptions (Breiter et al, 1996; MataixCols et al, 2004) specifically identifying rACC activation. One of these (Breiter et al, 1996) found rACC activation in OCD participants overall, whereas the other (Mataix-Cols et al, 2004) found rACC activation only in washers, and dACC activation in checkers. More recently, studies using task-based fMRI have demonstrated rACC-specific abnormalities in OCD patients. For example, Fitzgerald et al (2005) found significantly greater rACC hyperactivation in OCD patients compared with healthy controls after error commission on a flanker interference task that they hypothesized to result from an exaggerated affective response to errors. A later study in pediatric OCD patients by the same group (Fitzgerald et al, 2010) demonstrated errorrelated hyperactivation approaching statistical significance in a similar rACC region. Although these studies provide evidence of rACC dysfunction in OCD, they employed tasks designed primarily to examine cognitive interference processing-a function attributed largely to the dACC. Our findings expand on these prior studies to provide evidence of a failure to deactivate rACC in OCD by using an emotional interference task targeted to specifically probe rACC function (Whalen et al, 1998, 2006). Moreover, we have shown that this failed rACC deactivation is specific to OCD-related stimuli and does not generalize to emotionally salient stimuli unrelated to OCD.

The inability to disengage attention from emotionally salient stimuli in order to focus on a cognitive task (so-called 'emotional conflict') has been implicated in OCD (Cohen et al, 2003). Preliminary evidence suggests a specific role for the rACC in resolving emotional conflict, primarily through top-down inhibition of amygdalar activity (Etkin et al, 2006). Hence, failure to deactivate $\mathrm{rACC}$ in OCD may reflect sustained recruitment of this brain region to resolve emotional conflict and attend to task-relevant stimuli. We also found increased amygdalar activation in OCD participants compared with non-OCD participants in response to OCD-specific words, although at a more lenient statistical threshold, suggesting that rACC overcompensation in OCD patients may be insufficient to dampen amygdalar reactivity. This finding is consistent with a prior study of OCD patients identifying increased amygdalar activation to OCD-specific words, but not general emotion words, using a color-naming emotional Stroop task (van den Heuvel et al, 2005). However, in contrast to our findings, this study found no evidence of abnormal rACC activation in OCD patients, perhaps because of differences between the two studies in medication status and/or OCD severity. Although we did not find any significant between-group differences in connectivity between rACC and amygdala in response to OCD versus neutral words in our sample, further examination of rACC-amygdala connectivity in OCD patients may be informative to elucidate the interplay between these two critical brain regions.

Another way to conceptualize our finding of increased rACC activation in OCD patients is in the context of a set of brain regions that displayed increased reactivity to OCD-specific words. In addition to rACC, these regions included $\mathrm{mPFC}$, precuneus, and $\mathrm{pIPL}$-core regions associated with the brain's default mode network (DMN) 
Table 2 Between-Group Comparisons of BOLD Activation in Response to OCD-Specific Words Relative to Neutral Words

\begin{tabular}{|c|c|c|c|c|c|}
\hline \multirow[t]{2}{*}{$\begin{array}{l}\text { Brain region } \\
\text { (Brodmann area) }\end{array}$} & \multicolumn{3}{|c|}{$\begin{array}{c}\text { MNI } \\
\text { coordinates }\end{array}$} & \multirow[t]{2}{*}{ Z-score } & \multirow[t]{2}{*}{$\begin{array}{l}\text { Cluster } \\
\text { size }\end{array}$} \\
\hline & $\mathbf{x}$ & y & $\mathbf{z}$ & & \\
\hline \multicolumn{6}{|l|}{$O C D>$ non- $O C D$} \\
\hline \multirow[t]{2}{*}{ L medial superior frontal cortex $(10)$} & -14 & 62 & 2 & 4.79 & 678 \\
\hline & -4 & 58 & 20 & 4.73 & \\
\hline R rostral anterior cingulate cortex (32) & 10 & 50 & 10 & 4.1 & \\
\hline R precuneus $(23)$ & & -58 & 30 & 4.17 & 215 \\
\hline \multirow[t]{2}{*}{ L precuneus (23) } & -10 & -52 & 36 & 3.9 & \\
\hline & -6 & -52 & 26 & 3.59 & \\
\hline \multirow[t]{2}{*}{ L parieto-occipital cortex (39) } & $36-$ & -62 & 24 & 4.01 & 41 \\
\hline & $38-$ & -68 & 18 & 3.63 & \\
\hline L middle temporal cortex (39) & $-46-$ & -70 & 18 & 3.92 & 128 \\
\hline $\mathrm{L}$ angular cortex (39) & $-50-$ & -66 & 32 & 3.82 & \\
\hline L middle temporal cortex (39) & $-46-$ & -58 & 22 & 3.53 & \\
\hline $\mathrm{R}$ caudate & 18 & 14 & 10 & 3.87 & 137 \\
\hline \multirow[t]{2}{*}{ R putamen } & 26 & 16 & 6 & 3.63 & \\
\hline & 28 & 14 & -4 & 3.62 & \\
\hline R amygdala & 20 & & -10 & 3.86 & 29 \\
\hline L insula (48) & -26 & 16 & -8 & 3.84 & 24 \\
\hline R precuneus (23) & & -54 & 24 & 3.63 & 43 \\
\hline \multicolumn{6}{|l|}{ Non-OCD $>O C D$} \\
\hline None & & & & & \\
\hline
\end{tabular}

Abbreviations: BOLD, blood oxygen level dependent; L, left; MNI, Montreal Neurological Institute; $O C D$, obsessive-compulsive disorder; R, right.

(Buckner et al, 2008). Brain regions within the DMN have been repeatedly shown to deactivate during externally focused tasks and to activate during introspective cognitive processes such as autobiographical memory, self-referential thinking, and affective decision making (Buckner et al, 2008). In contrast to non-OCD participants who displayed robust rACC deactivation in response to OCD-specific words, participants with OCD showed rACC activation in response to OCD-specific words. Furthermore, no difference was observed in response to neutral words, suggesting that this abnormality is specific to emotionally salient stimuli. Consistent with our current finding, prior studies of OCD patients have demonstrated hyperactivity (primarily a failure to deactivate) among DMN brain regions, particularly ventromedial prefrontal cortex (vmPFC) and rACC, in response to cognitive paradigms examining uncertainty (Stern et al, 2013) and response to errors (Fitzgerald et al, 2005, 2010; Stern et al, 2011). In addition, connectivity analyses in OCD patients have shown reduced positive connectivity within DMN brain regions (Fitzgerald et al, 2010; Jang et al, 2010; Stern et al, 2012) as well as reduced negative connectivity between DMN and the frontoparietal network-a network of brain regions involved in directing attention toward externally focused tasks (Stern et al, 2012). These findings suggest that failure to deactivate rACC in OCD patients may be part of a network abnormality within DMN, and between DMN and other task-positive networks, leading to the inability to disengage from self-referential thought processes driven by OCD-specific stimuli.
Table 3 Within-Group Comparisons of BOLD Activation in Response to OCD-Specific Words Relative to Neutral Words

\begin{tabular}{|c|c|c|c|c|c|}
\hline \multirow[t]{2}{*}{$\begin{array}{l}\text { Brain region } \\
\text { (Brodmann area) }\end{array}$} & \multicolumn{3}{|c|}{$\begin{array}{c}\text { MNI } \\
\text { coordinates }\end{array}$} & \multirow[t]{2}{*}{ Z-score } & \multirow[t]{2}{*}{$\begin{array}{l}\text { Cluster } \\
\text { size }\end{array}$} \\
\hline & $\mathbf{x}$ & y & $\mathbf{z}$ & & \\
\hline \multicolumn{6}{|l|}{ Individuals with $O C D$} \\
\hline \multirow[t]{2}{*}{ L medial superior frontal cortex $(10)$} & -12 & 58 & 20 & 5.46 & 2093 \\
\hline & -14 & 62 & 2 & 5.09 & \\
\hline R medial superior frontal cortex $(10)$ & 6 & 58 & 28 & 5.08 & \\
\hline \multirow[t]{2}{*}{$L$ precuneus $(23)$} & -6 & -52 & 26 & 4.35 & 478 \\
\hline & -6 & -60 & 34 & 4.27 & \\
\hline R precuneus (23) & & -58 & 30 & 3.56 & \\
\hline L inferior frontal cortex (45) & -44 & 22 & 6 & 4.33 & 72 \\
\hline \multirow[t]{3}{*}{ L middle temporal cortex (39) } & -50 & -66 & 32 & 4.32 & 260 \\
\hline & -44 & -58 & 24 & 4.24 & \\
\hline & -40 & & 34 & 3.29 & \\
\hline \multirow[t]{2}{*}{$\mathrm{R}$ inferior frontal cortex (45) } & 52 & 24 & 0 & 3.85 & 50 \\
\hline & -40 & 28 & -10 & 3.75 & 25 \\
\hline R medial superior frontal cortex (9) & 12 & 36 & 54 & 3.74 & 27 \\
\hline$L$ insula $(48)$ & -28 & 16 & -18 & 3.73 & 21 \\
\hline \multicolumn{6}{|l|}{ Individuals without $O C D$} \\
\hline None & & & & & \\
\hline
\end{tabular}

Abbreviations: BOLD, blood oxygen level dependent; L, left; MNI, Montreal Neurological Institute; OCD, obsessive-compulsive disorder; R, right.

Contrary to our a priori hypotheses, we failed to find between-group differences in Gln/Glu, Gln, or Glu levels in the rACC, and we found no association between Gln/Glu and rACC BOLD activation in the OCD group. Among the 10 prior MRS studies known to us comparing ACC levels of Glu-related metabolites in OCD patients and healthy controls (Bedard and Chantal, 2011; Ebert et al, 1997; Gnanavel et al, 2013; Lázaro et al, 2012; O'Neill et al, 2012; Rosenberg et al, 2004; Simpson et al, 2012; Starck et al, 2008; Yücel et al, 2007, 2008), only 2 studies found decreased levels of Glx (Rosenberg et al, 2004; Yücel et al, 2008), with one of these finding reduced Glx only in females with OCD (Yücel et al, 2008), and one found increased Glx (Gnanavel et al, 2013). These discrepancies may be because of small sample sizes and methodological inconsistencies or perhaps the few existing positive findings represent false positives. Alternatively, glutamatergic abnormalities might be restricted to certain subgroups of OCD patients defined by genetically mediated factors (Arnold et al, 2009; Pauls et al, 2014). Yet another possibility is that abnormal levels of Glu-related metabolites may exist only in the early course of OCD. The first reports of abnormal Glx levels in OCD were in pediatric patients (Rosenberg et al, 2000, 2004) and most subsequent studies in adult OCD (Bartha et al, 1998; Bedard and Chantal, 2011; Ebert et al, 1997; Lázaro et al, 2012; Simpson et al, 2012; Starck et al, 2008; Whiteside et al, 2012; Yücel et al, 2007), with some exceptions (Gnanavel et al, 2013; Whiteside et al, 2006; Yücel et al, 2008), have found no abnormalities in Glx or Glu levels. One plausible explanation is that glutamatergic abnormalities in early OCD may lead to compensatory changes in receptor profile and synaptic strength that subsequently alter neuronal activation-a 
homeostatic plasticity well documented in glutamatergic neurons (Turrigiano, 2008). Once a new activity 'set-point' is established, glutamatergic neurotransmission may stabilize and levels of Glu-related metabolites may normalize. Although this theory is speculative, it warrants further exploration of Glu and Gln levels at various time points in longitudinal ${ }^{1} \mathrm{H}$-MRS studies of OCD patients.

We believe that our study is noteworthy for several reasons. First, we found convincing evidence of rACC dysfunction specific to OCD-related stimuli in participants with OCD using a novel OCD-modified version of the ecStroop task designed specifically to probe this region. Second, this is the first study of individuals with OCD to use $J$-resolved ${ }^{1} \mathrm{H}$-MRS to quantify brain levels of Glu and Gln separately, providing a more accurate assessment of glutamatergic activity. Third, to our knowledge, this is the largest sample of OCD participants in which Glu-related metabolites were examined. Therefore, we believe that our study offers additional evidence arguing against glutamatergic dysfunction in the rACC in adult OCD.

We also acknowledge several limitations. First, we included participants with OCD who were on a stable medication regimen. Although post hoc analyses showed no significant influence of medications on either rACC BOLD or Glu-related measures, we acknowledge that the sample of unmedicated OCD participants was small and our analyses may have been underpowered to detect such an effect. Similarly, we also acknowledge that many OCD participants had been receiving outpatient behavioral therapy before admission that may have affected our findings. Importantly, however, as reflected in the entry criteria and participant characterization information, all OCD participants had substantial symptom severity at the time of scanning. Second, we included comorbid mental disorders, including depression and anxiety disorders, that may have affected our results. Third, we expressed Glu and Gln levels as ratios to $\mathrm{tCr}-\mathrm{a}$ method that presumes no between-group $\mathrm{tCr}$ differences (Öngür et al, 2009). However, prior MRS studies of OCD patients have suggested no significant $\mathrm{tCr}$ abnormalities (Brennan et al, 2013) and we found no between-group differences in the $\mathrm{tCr} /$ total signal ratio in our sample, indicating that our use of internal tCr normalization is valid. Fourth, we did not collect data on smoking habits in our sample. Given the possibility that cerebral Glu levels may be affected by nicotine use (Licata and Renshaw, 2010), we cannot rule out the potential confounding effects of nicotine use on our findings. Finally, the OCD patients included in this study were being treated in an intensive residential treatment program and thus likely represent a severe, treatment-refractory subgroup (Brennan et al, 2014). Therefore, our findings may not be reflective of the broader OCD population.

In summary, comparing OCD patients with individuals without OCD on an OCD-modified ecStroop task, we found significantly reduced $\mathrm{rACC}$ BOLD deactivation that was not associated with abnormal rACC Gln/Glu ratios. Failure to deactivate rACC in OCD may represent an exaggerated response aimed at resolving emotional conflict or may reflect a broader inability to disengage the DMN when patients are exposed to self-relevant emotionally salient stimuli. Future fMRI studies combining both task-related and functional connectivity analyses are necessary to more fully understand how this inability to deactivate rACC contributes to OCD and to examine the impact of medication and behavioral interventions on rACC deactivation to determine whether this is a state marker that normalizes with successful treatment.

\section{FUNDING AND DISCLOSURE}

Dr Brennan has received research grant support from Eli Lilly and Transcept Pharmaceuticals. Dr Hudson has received consulting fees from Genentech, HealthCore, Roche, and Shire; has received research grant support from Genentech, Otsuka, and Shire; and has received compensation for expert legal testimony from various law firms. Dr Pope has received compensation for expert legal testimony from various law firms. Over the past 3 years, Dr Rauch has received compensation from NIMH, University of North Carolina, University of Cincinnati, The Center for Addiction and Mental Health-Toronto, Oxford University Press, APPI, Elsevier Publishing, The American Psychiatric Foundation, WGBH, and Hall -Mercer (in addition to his primary employer, Partners Healthcare). The authors declare no conflict of interest.

\section{ACKNOWLEDGMENTS}

We thank Diane Davey, Brittany Mathes, Jordan Cattie, and Marie-Christine Andre for assistance with screening and recruitment of participants. We also thank the OCD patients who participated in this study. This work was funded in part by grant K23-MH092397 from the National Institute of Mental Health (to BPB), the Sidney R Baer, Jr Foundation through a NARSAD Young Investigator Award (to BPB), Grant K23-MH104515 from the National Institute of Mental Health (to JTB), and the David Judah Fund at Massachusetts General Hospital (to MAJ).

\section{REFERENCES}

Adler CM, McDonough-Ryan P, Sax KW, Holland SK, Arndt S, Strakowski SM (2000). fMRI of neuronal activation with symptom provocation in unmedicated patients with obsessive compulsive disorder. J Psychiatr Res 34: 317-324.

Alexander GE, DeLong MR, Strick PL (1986). Parallel organization of functionally segregated circuits linking basal ganglia and cortex. Annu Rev Neurosci 9: 357-381.

Arnold PD, Macmaster FP, Richter MA, Hanna GL, Sicard T, Burroughs E et al (2009). Glutamate receptor gene (GRIN2B) associated with reduced anterior cingulate glutamatergic concentration in pediatric obsessive-compulsive disorder. Psychiatry Res 172: 136-139.

Bartha R, Stein MB, Williamson PC, Drost DJ, Neufeld RW, Carr TJ et al (1998). A short echo $1 \mathrm{H}$ spectroscopy and volumetric MRI study of the corpus striatum in patients with obsessivecompulsive disorder and comparison subjects. Am J Psychiatry 155: 1584-1591.

Baxter LR Jr., Phelps ME, Mazziotta JC, Guze BH, Schwartz JM, Selin CE (1987). Local cerebral glucose metabolic rates in obsessive-compulsive disorder. A comparison with rates in unipolar depression and in normal controls. Arch Gen Psychiatry 44: 211-218.

Bedard MJ, Chantal S (2011). Brain magnetic resonance spectroscopy in obsessive-compulsive disorder: the importance of 
considering subclinical symptoms of anxiety and depression. Psychiatry Res 192: 45-54.

Breiter HC, Rauch SL, Kwong KK, Baker JR, Weisskoff RM, Kennedy DN et al (1996). Functional magnetic resonance imaging of symptom provocation in obsessive-compulsive disorder. Arch Gen Psychiatry 53: 595-606.

Brennan BP, Hudson JI, Jensen JE, McCarthy J, Roberts JL, Prescot AP et al (2010). Rapid enhancement of glutamatergic neurotransmission in bipolar depression following treatment with riluzole. Neuropsychopharmacology 35: 834-846.

Brennan BP, Lee C, Elias JA, Crosby JM, Mathes BM, Andre MC et al (2014). Intensive residential treatment for severe obsessivecompulsive disorder: characterizing treatment course and predictors of response. J Psychiatr Res 56: 98-105.

Brennan BP, Rauch SL, Jensen JE, Pope HG Jr. (2013). A critical review of magnetic resonance spectroscopy studies of obsessivecompulsive disorder. Biol Psychiatry 73: 24-31.

Britton JC, Gold AL, Deckersbach T, Rauch SL (2009). Functional MRI study of specific animal phobia using an event-related emotional counting stroop paradigm. Depress Anxiety 26: 796-805.

Buckner RL, Andrews-Hanna JR, Schacter DL (2008). The brain's default network: anatomy, function, and relevance to disease. Ann NY Acad Sci 1124: 1-38.

Bush G, Luu P, Posner MI (2000). Cognitive and emotional influences in anterior cingulate cortex. Trends Cogn Sci 4: 215-222.

Cohen Y, Lachenmeyer JR, Springer C (2003). Anxiety and selective attention in obsessive-compulsive disorder. Behav Res Ther 41: 1311-1323.

Ebert D, Speck O, Konig A, Berger M, Hennig J, Hohagen F (1997). $1 \mathrm{H}$-magnetic resonance spectroscopy in obsessive-compulsive disorder: evidence for neuronal loss in the cingulate gyrus and the right striatum. Psychiatry Res 74: 173-176.

Etkin A, Egner T, Peraza DM, Kandel ER, Hirsch J (2006). Resolving emotional conflict: a role for the rostral anterior cingulate cortex in modulating activity in the amygdala. Neuron 51: $871-882$.

Fitzgerald KD, Stern ER, Angstadt M, Nicholson-Muth KC, Maynor MR, Welsh RC et al (2010). Altered function and connectivity of the medial frontal cortex in pediatric obsessivecompulsive disorder. Biol Psychiatry 68: 1039-1047.

Fitzgerald KD, Welsh RC, Gehring WJ, Abelson JL, Himle JA, Liberzon I et al (2005). Error-related hyperactivity of the anterior cingulate cortex in obsessive-compulsive disorder. Biol Psychiatry 57: 287-294.

Gnanavel S, Sharan P, Khandelwal S, Sharma U, Jagannathan NR (2013). Neurochemicals measured by H-MR spectroscopy: putative vulnerability biomarkers for obsessive compulsive disorder. MAGMA 27: 407-417.

Igarashi H, Kwee IL, Nakada T, Katayama Y, Terashi A (2001). 1H magnetic resonance spectroscopic imaging of permanent focal cerebral ischemia in rat: longitudinal metabolic changes in ischemic core and rim. Brain Res 907: 208-221.

Iltis I, Koski DM, Eberly LE, Nelson CD, Deelchand DK, Valette J et al (2009). Neurochemical changes in the rat prefrontal cortex following acute phencyclidine treatment: an in vivo localized (1) $\mathrm{H}$ MRS study. NMR Biomed 22: 737-744.

Jang JH, Kim JH, Jung WH, Choi JS, Jung MH, Lee JM et al (2010). Functional connectivity in fronto-subcortical circuitry during the resting state in obsessive-compulsive disorder. Neurosci Lett 474: $158-162$.

Jensen JE, Licata SC, Öngür D, Friedman SD, Prescot AP, Henry ME et al (2009). Quantification of J-resolved proton spectra in two-dimensions with LCModel using GAMMAsimulated basis sets at 4 Tesla. NMR Biomed 22: 762-769.

Landeros-Weisenberger A, Bloch $\mathrm{MH}$, Kelmendi B, Wegner R, Nudel J, Dombrowski P et al (2010). Dimensional predictors of response to SRI pharmacotherapy in obsessive-compulsive disorder. J Affect Disord 121: 175-179.

Lázaro L, Bargallo N, Andres S, Falcon C, Morer A, Junque C et al (2012). Proton magnetic resonance spectroscopy in pediatric obsessive-compulsive disorder: longitudinal study before and after treatment. Psychiatry Res 30: 17-24.

Licata SC, Renshaw PF (2010). Neurochemistry of drug action: insights from proton magnetic resonance spectroscopic imaging and their relevance to addiction. Ann NY Acad Sci 1187: $148-171$.

Machlin SR, Harris GJ, Pearlson GD, Hoehn-Saric R, Jeffery P, Camargo EE (1991). Elevated medial-frontal cerebral blood flow in obsessive-compulsive patients: a SPECT study. Am J Psychiatry 148: $1240-1242$.

Maldjian JA, Laurienti PJ, Kraft RA, Burdette JH (2003). An automated method for neuroanatomic and cytoarchitectonic atlas-based interrogation of fMRI data sets. Neuroimage 19: 1233-1239.

Mataix-Cols D, Wooderson S, Lawrence N, Brammer MJ, Speckens A, Phillips ML (2004). Distinct neural correlates of washing, checking, and hoarding symptom dimensions in obsessive-compulsive disorder. Arch Gen Psychiatry 61: 564-576.

McLaren DG, Ries ML, Xu G, Johnson SC (2012). A generalized form of context-dependent psychophysiological interactions (gPPI): a comparison to standard approaches. Neuroimage 61: $1277-1286$.

O'Neill J, Piacentini JC, Chang S, Levitt JG, Rozenman M, Bergman L et al (2012). MRSI correlates of cognitive-behavioral therapy in pediatric obsessive-compulsive disorder. Prog Neuropsychopharmacol Biol Psychiatry 36: 161-168.

O'Reilly JX, Woolrich MW, Behrens TE, Smith SM, Johansen-Berg H (2012). Tools of the trade: psychophysiological interactions and functional connectivity. Soc Cogn Affect Neurosci 7: 604-609.

Öngür D, Haddad S, Prescot AP, Jensen JE, Siburian R, Cohen BM et al (2011). Relationship between genetic variation in the glutaminase gene GLS1 and brain glutamine/glutamate ratio measured in vivo. Biol Psychiatry 70: 169-174.

Öngür D, Jensen JE, Prescot AP, Stork C, Lundy M, Cohen BM et al (2008). Abnormal glutamatergic neurotransmission and neuronal-glial interactions in acute mania. Biol Psychiatry 64: 718-726.

Öngür D, Prescot AP, Jensen JE, Cohen BM, Renshaw PF (2009). Creatine abnormalities in schizophrenia and bipolar disorder. Psychiatry Res 172: 44-48.

Pauls DL, Abramovitch A, Rauch SL, Geller DA (2014). Obsessivecompulsive disorder: an integrative genetic and neurobiological perspective. Nat Rev Neurosci 15: 410-424.

Perani D, Colombo C, Bressi S, Bonfanti A, Grassi F, Scarone S et al (1995). [18F]FDG PET study in obsessive-compulsive disorder. A clinical/metabolic correlation study after treatment. $\mathrm{Br} J$ Psychiatry 166: 244-250.

Pittenger C, Bloch MH, Williams K (2011). Glutamate abnormalities in obsessive compulsive disorder: neurobiology, pathophysiology, and treatment. Pharmacol Ther 132: 314-332.

Provencher SW (1993). Estimation of metabolite concentrations from localized in vivo proton NMR spectra. Magn Reson Med 30: 672-679.

Rauch SL, Jenike MA, Alpert NM, Baer L, Breiter HC, Savage CR et al (1994). Regional cerebral blood flow measured during symptom provocation in obsessive-compulsive disorder using oxygen 15-labeled carbon dioxide and positron emission tomography. Arch Gen Psychiatry 51: 62-70.

Rauch SL, Shin LM, Segal E, Pitman RK, Carson MA, McMullin K et al (2003). Selectively reduced regional cortical volumes in posttraumatic stress disorder. Neuroreport 14: 913-916.

Rosenberg DR, MacMaster FP, Keshavan MS, Fitzgerald KD, Stewart CM, Moore GJ (2000). Decrease in caudate glutamatergic concentrations in pediatric obsessive-compulsive disorder 
patients taking paroxetine. J Am Acad Child Adolesc Psychiatry 39: 1096-1103.

Rosenberg DR, Mirza Y, Russell A, Tang J, Smith JM, Banerjee SP et al (2004). Reduced anterior cingulate glutamatergic concentrations in childhood OCD and major depression versus healthy controls. J Am Acad Child Adolesc Psychiatry 43: 1146-1153.

Saxena S, Rauch SL (2000). Functional neuroimaging and the neuroanatomy of obsessive-compulsive disorder. Psychiatr Clin North Am 23: 563-586.

Shin LM, Whalen PJ, Pitman RK, Bush G, Macklin ML, Lasko NB et al (2001). An fMRI study of anterior cingulate function in posttraumatic stress disorder. Biol Psychiatry 50: 932-942.

Sibson NR, Shen J, Mason GF, Rothman DL, Behar KL, Shulman RG (1998). Functional energy metabolism: in vivo 13C-NMR spectroscopy evidence for coupling of cerebral glucose consumption and glutamatergic neuronalactivity. Dev Neurosci 20: $321-330$

Simpson HB, Shungu DC, Bender J Jr., Mao X, Xu X, Slifstein M et al (2012). Investigation of cortical glutamate-glutamine and gamma-aminobutyric acid in obsessive-compulsive disorder by proton magnetic resonance spectroscopy. Neuropsychopharmacology 37: 2684-2692.

Starck G, Ljungberg M, Nilsson M, Jonsson L, Lundberg S, Ivarsson $\mathrm{T}$ et al (2008). A $1 \mathrm{H}$ magnetic resonance spectroscopy study in adults with obsessive compulsive disorder: relationship between metabolite concentrations and symptom severity. J Neural Transm 115: 1051-1062.

Stern ER, Fitzgerald KD, Welsh RC, Abelson JL, Taylor SF (2012). Resting-state functional connectivity between fronto-parietal and default mode networks in obsessive-compulsive disorder. PLoS One 7: e36356.

Stern ER, Welsh RC, Fitzgerald KD, Gehring WJ, Lister JJ, Himle JA et al (2011). Hyperactive error responses and altered connectivity in ventromedial and frontoinsular cortices in obsessivecompulsive disorder. Biol Psychiatry 69: 583-591.

Stern ER, Welsh RC, Gonzalez R, Fitzgerald KD, Abelson JL, Taylor SF (2013). Subjective uncertainty and limbic hyperactivation in obsessive-compulsive disorder. Hum Brain Mapp 34: 1956-1970.
Swedo SE, Schapiro MB, Grady CL, Cheslow DL, Leonard HL, Kumar A et al (1989). Cerebral glucose metabolism in childhoodonset obsessive-compulsive disorder. Arch Gen Psychiatry 46: 518-523.

Turrigiano GG (2008). The self-tuning neuron: synaptic scaling of excitatory synapses. Cell 135: 422-435.

Tzourio-Mazoyer N, Landeau B, Papathanassiou D, Crivello F, Etard O, Delcroix N et al (2002). Automated anatomical labeling of activations in SPM using a macroscopic anatomical parcellation of the MNI MRI single-subject brain. Neuroimage 15: 273-289.

van den Heuvel OA, Veltman DJ, Groenewegen HJ, Witter MP, Merkelbach J, Cath DC et al (2005). Disorder-specific neuroanatomical correlates of attentional bias in obsessive-compulsive disorder, panic disorder, and hypochondriasis. Arch Gen Psychiatry 62: 922-933.

Vogt BA, Nimchinsky EA, Vogt LJ, Hof PR (1995). Human cingulate cortex: surface features, flat maps, and cytoarchitecture. J Comp Neurol 359: 490-506.

Whalen PJ, Bush G, McNally RJ, Wilhelm S, McInerney SC, Jenike MA et al (1998). The emotional counting Stroop paradigm: a functional magnetic resonance imaging probe of the anterior cingulate affective division. Biol Psychiatry 44: 1219-1228.

Whalen PJ, Bush G, Shin LM, Rauch SL (2006). The emotional counting Stroop: a task for assessing emotional interference during brain imaging. Nat Protoc 1: 293-296.

Whiteside SP, Abramowitz JS, Port JD (2012). The effect of behavior therapy on caudate $\mathrm{N}$-acetyl-l-aspartic acid in adults with obsessive-compulsive disorder. Psychiatry Res 30: 10-16.

Whiteside SP, Port JD, Deacon BJ, Abramowitz JS (2006). A magnetic resonance spectroscopy investigation of obsessivecompulsive disorder and anxiety. Psychiatry Res 146: 137-147.

Yücel M, Harrison BJ, Wood SJ, Fornito A, Wellard RM, Pujol J et al (2007). Functional and biochemical alterations of the medial frontal cortex in obsessive-compulsive disorder. Arch Gen Psychiatry 64: 946-955.

Yücel M, Wood SJ, Wellard RM, Harrison BJ, Fornito A, Pujol J et al (2008). Anterior cingulate glutamate-glutamine levels predict symptom severity in women with obsessive-compulsive disorder. Aust N Z J Psychiatry 42: 467-477.

Supplementary Information accompanies the paper on the Neuropsychopharmacology website (http://www.nature.com/npp) 